\title{
Improved Haze Removal of Underwater Images using Particle Swarm Optimization
}

\author{
Shriya Sharma \\ B Tech, CSE \\ Computer SCI \& Engg Department \\ Guru Nanak Dev University, Amritsar
}

\author{
Sakshi Bhalla \\ B Tech, CSE \\ Computer SCI \& Engg Department \\ Shaheed Bhagat Singh Tech \\ Campus Ferozpur
}

\begin{abstract}
The main objective of fog removal algorithm is to estimate the airlight map for the given image and then perform the necessary operations on the image in order to overcome the fog in the image and enhance the quality of the image. The dark channel prior method of fog removal is more suitable and time-saving in real-time systems. In this paper, an efficient approach for fog removal of foggy images based on the combination of dark channel prior and genetic algorithm is presented. It is found that the proposed method is more suitable for obtaining the better quality of the image than the most of the existing methods.
\end{abstract}

\section{Keywords}

Dark Channel Prior, Genetic Algorithm, Transmission Map

\section{INTRODUCTION}

Visibility restoration [1] refers to different ways that help to reduce and remove the degradation which occurs when a digital image is taken. The image suffers from degradation due to various reasons such as relative object-camera motion, blur due to camera misfocus, relative atmospheric turbulence etc. The main cause of image degradation is due to bad weather conditions such as fog, haze, rain and snow . During Fog, when we take an image using a camera then the light gets scattered before reaching the camera due to some impurities in the atmosphere. Due to this, automatic monitoring system, outdoor recognition system and intelligent transportation system are badly affected. Scattering of light is caused by two fundamental phenomena such as attenuation and airlight. By using haze removal algorithms, we can enhance the stability and robustness of the visual system. Removal of haze is a difficult task because fog depends upon the unknown scene depth information. Fog effect is defined as event of distance between camera and object. Hence removal of fog requires the estimation of airlight map or depth map. The haze removal techniques can be classified into two categories: image enhancement and image restoration. Image enhancement doesn't include the reason why fog degrades image quality. This technique enhances the contrast of haze image but it leads to loss of information in image. Image restoration studies the physical procedure of imaging in fog.
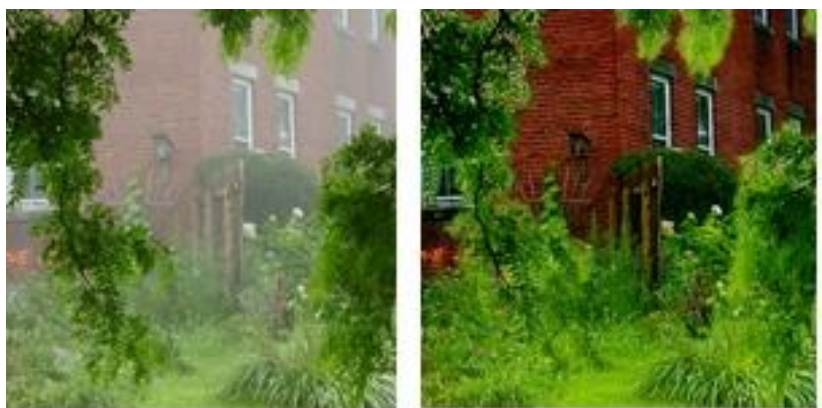

Fig 1: (a) Original image (b) Processed image

After observing degradation style of fog, image will undoubtedly be established. At last, the degradation process is used to produce the fog free image.

\section{HAZE REMOVAL TECHNIQUES}

As a way to remove the fog effect from the image various fog removal methods are used. By eliminating fog, visibility of the image can be increased and the color shift due to the airlight can even be verified to a large extent. Many of the most commonly used methods are as under.

\subsection{DARK CHANNEL PRIOR}

Dark channel prior [1] is used for the estimation of atmospheric light in the dehazed image to get the more proper result. This technique is utilized for non-sky patches, as at least one color channel has low intensity at few pixels. The low intensity in the dark channel is predominantly due to three components:-

- Colourful items or surfaces.

- Shadows(shadows of car, buildings etc)

- Dark items or surfaces(dark tree trunk, stone )

As the outdoor images are genrally full of shadows and colorful, the dark channels of images will be dark. Because of fog (airlight), a haze image is brighter than its image without haze. So it can be said that dark channel of haze image will have larger intensity in region with lot of haze. Hence, the dark channel's intensity is a rough approximation of the thickness of haze. In dark channel prior one also utilize pre and post processing steps for getting best results. In post processing steps one utilize soft matting or bilateral filtering etc. Let input image be $J(x)$, foggy image be $I(x), t(x)$ is the transmission of the medium. The image attenuation which is due to fog can be expressed as:

$$
I_{\text {att }}(x)=J(x) t(x) \ldots \ldots(1)
$$

The fog effect is Airlight effect expressed as: 


$$
I_{\text {airlight }}(x)=A(1-t(x)) \ldots \ldots(2)
$$

Dark channel for an arbitrary image $\mathbf{J}$, $\mathbf{J}$ dark is defined as:

$$
J^{\operatorname{dark}}(x)=\frac{\min }{y \in \Omega(x)}\left(\min J^{C}(Y)\right) \ldots \ldots(3)
$$

In this $\mathbf{J}^{\mathrm{c}}$ is the color image comprises of RGB components, $\Omega(x)$ represents a local patch which has origin at $\mathrm{x}$. The lower intensity of dark channels is attributed due to shadows of the images, saturated color objects and dark objects.

Subsequent to dark channel prior, one need to estimate transmission $\mathrm{t}(\mathrm{x})$ for proceeding with the solution. Another supposition needed is that let Atmospheric light $\mathrm{A}$ is also known. One normalize (4) by dividing both sides by A:

$\frac{I^{c}}{A^{c}}(x)=\mathrm{t}(\mathrm{x}) \frac{J^{c}}{A^{c}}(x)+1-\mathrm{t}(\mathrm{x})$

\section{GENETIC ALGORITHM}

Genetic algorithms have now been applied almost exclusively to single-attribute 1 problems. But a cautious look at many real-world GA applications reveals that the objective functions are actually multi attribute. Typically, the GA user some ad-hoc function of the multiple attributes to yield a scalar function. Often-seen tools for combining multiple attributes are constraints, with associated thresholds and penalty functions, and weights for linear combinations of attribute values. But penalties and weights have demonstrated to be problematic. The GA solution is generally very sensitive to small changes in the penalty function coefficients and weighting factors.

\section{LITERATURE SURVEY}

Podzimek, Josef [1] discussed that droplet imprints in thin gelatin layers have already been employed for the investigation of coarse particles embedded in fog (haze) droplets compared to interstitial particles. Droplet samples were taken in less polluted elements of Missouri and Illinois and also in more polluted marine-urban environment in Italy. Considerable differences in particle size distribution and particle location within the droplets have now been present in samples collected on four sampling sites. The deduced relationships could be requested the description of the aerosol scavenging process in fogs. The pollution of haze droplets might be linked to the droplet mean volume radius and may be determined by temperature and on atmospheric humidity. An orientative study of the deposited particle composition indicated important relationships between coarse particles of different origin like soil minerals, industrial and urban pollutants, sea salt. Chu, Chao-Tsung, and Ming-Sui Lee [2] proposed A content adaptive method for single image dehazing. Considering that the degradation level suffering from haze relates to the depth of the scene and pixels in each specific area of the image like trees, buildings and other objects generally have similar depth to the camera, they assumed that the degradation level suffering from haze of every region is exactly the same That's, the transmission in each region ought to be similar as well. Based with this assumption, each input image is segmented into different regions and transmission is estimated for every region followed closely by refinement by soft matting. Consequently, the hazy images may be successfully recovered. The experimental results demonstrate that the proposed method performs satisfactorily. Ancuti, Codruta O. et al. [3] introduced a new approach to displace just one image degraded by atmospheric phenomena such as for instance fog or haze. The presented algorithm provides for fast identification of hazy elements of an image without using expensive optimization and refinement procedures. Through the use of just one per pixel operation on the initial image, they produced a 'semi-inverse' of the image. On the basis of the hue disparity between the initial image and its semiinverse, they certainly were then able to recognize hazy regions on a per pixel basis. This enables for an easy estimation of the airlight constant and the transmission map. Their approach is dependent on a comprehensive study on a sizable data group of images, and validated predicated on a metric that measures the contrast but additionally the structural changes. The algorithm is straightforward and performs faster than existing strategies while yielding comparative and better still results. In addition they provided a comparative evaluation against other recent single image dehazing methods, demonstrating the efficiency and utility of the approach. Zhang, Yong-Qin et al. [4] proposed a new effective algorithm for visibility enhancement from just one gray or color image. Because it can be viewed that the haze mainly concentrates in a single element of the multilayer image, the haze-free image is reconstructed through haze layer estimation on the basis of the image filtering approach using both low-rank technique and the overlap averaging scheme. By utilizing parallel analysis with Monte Carlo simulation from the coarse atmospheric veil by the median filter, the refined smooth haze layer is acquired with both less texture and retaining depth changes. With the dark channel prior, the normalized transmission coefficient is calculated to displace fogless image. Experimental results reveal that the proposed algorithm is an easier and efficient method for clarity improvement and contrast enhancement from just one foggy image. Moreover, it may be comparable with the state-of-theart methods, and even has better results than them. Xiao, Chunxia, and Jiajia Gan [5] proposed a novel fast dehazing method from single image centered on filtering. The fundamental idea would be to compute a precise atmosphere veil that's not just smoother, but additionally respect with depth information of the underlying image. They firstly obtained an original atmosphere scattering light through median filtering, then refine it by guided joint bilateral filtering to generate a novel atmosphere veil which removes the abundant texture information and recovers the depth edge information. Finally, they solved the scene radiance utilizing the atmosphere attenuation model. In contrast to exiting state of the art dehazing methods, their method might get a much better dehazing effect at distant scene and places where depth changes abruptly. Their method is fast with linear complexity in the amount of pixels of the input image; furthermore, as their method could be performed in parallel, thus it may be further accelerated using GPU, making this process applicable for real-time requirement. Xie, Bin et al. [6] presented a new approach to get rid of haze from surveillance video sequences. The approach first extracts the back ground image through frames differential method, and then estimates the atmospheric light and transmission map from the back ground image, finally renders haze-free video in line with the haze image model. The key advantageous asset of the proposed approach is its speed since this method adopts a 'universal strategy' that's applying exactly the same atmospheric light and an individual pseudo-transmission map to some video frames. Experiments and performance analysis demonstrate that the good haze-free video may be produced effectively and efficiently. Kim, Eun-Kyoung et al. [7] proposed the hardware architecture of bilateral filter that may smooth image while preserving edges for the real-time image processing. Bilateral 
filter is nonlinear mixture of nearby image values. As the full time complexity of bilateral filter is $\mathrm{O}$ (total pixel size $*$ filter kernel size 2), the real-time processing is difficult. They reduced the full time complexity of bilateral filter using parallel architecture to attain real-time performance. Ding, Meng, and RuoFeng Tong [8] presented a hierarchical method of accelerate dark channel based image dehazing. The core of the approach is an efficient scheme for solving the soft matting problem involved with image dehazing, using adaptively subdivided quad trees built-in image space. Acceleration is accomplished by transforming the situation of solving a $N$-variable linear system required in soft matting, to an issue of solving a much smaller $m$-variable linear system, where $N$ is the amount of pixels and $m$ is the amount of the corners in the quad tree. Their approach significantly reduces both space and time cost while still maintains visual fidelity, and largely extends the practicability of dark channel based image dehazing to deal with large images. Xue, Yungang et al. [9] presented its parallel implementation and optimization on the basis of the GPU, which greatly reduces the execution time. They firstly implemented the essential parallel program, and then optimize it to acquire performance acceleration with increased than 20 times. They introduced the technique of "guide image filter" to Haze Removal Using Dark Channel Prior, rather than "soft matting" method, which largely reduces memory requirements and the computation complexity of the initial algorithm. While paralleling and optimizing the algorithm, they improved some areas of the initial serial program or basic parallel program based on the characteristics of several steps, and proposed a new approach to selecting atmospheric light and a new parallel approach to calculating accumulative sum with keeping intermediate results, which reduce steadily the computation complexity of counterpart and boost the parallel degree. Lan, Xia et al. [10] proposed a three-stage algorithm for haze removal, considering sensor blur and noise. In the initial stage, they preprocessed the degraded image and get rid of the blur/noise interference to estimate the hazy image. In the next stage, they estimated the transmission and atmospheric light by the dark channel prior method. In the next stage, a regularized method is proposed to recuperate the underlying image. Experimental results with both simulated and real data demonstrate that the proposed algorithm works well, centered on both visual effect and quantitative assessment. Ogorodnikov, B. I., and V. E. Khan [11] presented the outcome of observations of the impact of fog and haze on the operation of filtering units for the sampling of radioactive aerosols along the way of monitoring radiation conditions. It's revealed that the performance of installations decreases in the event of the forming of fog and mist. This really is related to the settling of finest water droplets on the fibers and with the clogging of the filter. Because the fog disappears, the droplets evaporate from the filtering fibers and the performance of the machine increases. In the event of fog and haze through the night in November 2012, the performance of AURA-02.11 installation decreased almost by three times as in contrast to the first one. To lessen the negative impact of fog and haze on the operation of units, it's reasonable to utilize the filters made from hydrophobic fibers Guo, Fan et al. [12] presented two haze removal algorithms for single image centered on haziness analysis. One algorithm regards haze because the veil layer, and another takes haze because the transmission. The former uses the illumination component image obtained by retinex algorithm and the depth information of the initial image to get rid of the veil layer. The latter employs guided filter to acquire the refined haze transmission and separates it from the first image. The key benefits of the proposed methods are that no user interaction will become necessary and the computing speed is relatively fast. A comparative study and quantitative evaluation with some main existing algorithms demonstrate that similar even higher quality results may be obtained by the proposed methods. At the top of haze removal, several applications of the haze transmission including image refocusing, haze simulation, relighting and 2dimensional (2D) to 3-dimensional (3D) stereoscopic conversion may also be implemented. Galdran, Adrian et al. [13] proposed to give a well-known perception-inspired variational framework [1] for the job of single image dehazing. The key modification consists on the replacement of the worthiness utilized by this framework for the greyworld hypothesis by an estimation of the mean of the clean image. This permits us to devise a variational method that will require no estimate of the depth structure of the scene, performing a spatially-variant contrast enhancement that effectively removes haze from a long way away regions. Experimental results reveal that their method competes well with other state-of-the-art methods in typical benchmark images, while outperforming current image dehazing methods in tougher scenarios. Zhang, Jun, and Shiqiang $\mathrm{Hu}$ [14] proposed a graphic processing unit (GPU)-accelerated realtime image enhancing method to get rid of haze from just one hazy input image. The inspiration of this process is a new pixel-level optimal de-hazing criterion proposed to mix an electronic hazy-free candidate image sequence into one last single hazy-free image. This image sequence is estimated from the input hazy image by exhausting all possible discretely sampled scene depth values. The key advantageous asset of proposed method may be the single pixel independently computing fashion. Its computing at a single pixel position is independent of others. Based with this property, it's straightforward to implement the proposed method with fully parallel GPU acceleration. The sufficient experimentations on various scenes indicate that the proposed method can process a sizable hazy image with one megapixel to visually moderate haze-free result at an interest rate of 80 frames/s. Moreover, the proposed method can also be less afflicted with the nonuniform illumination in comparison to previous methods. Liu, Qian et al. [15] proposed an intuitive and effective method, called the depth-based contrast stretching transform (DCST), to simultaneously obtain clear visibility and enhance contrast of just one haze gray image. compared to the image recovered via the physical-based model. Experiments demonstrate that images stretched by the DCST have excellent visibility and contrast in contrast to a couple of existing algorithms. Compelling performance can also be presented by comparing the proposed method with other representative methods in the applying of local feature detection. Gadnayak, Khitish Kumar et al. [17] dedicated to the haze removal process by thinking about the HSI color type of a picture as opposed to RGB color space. In the HSI color model, the saturation component describes the contrast of an The DCST stretches the contrast of haze images on the basis of the coarse depth layers of scenes. Their method is straightforward and almost real-time and could be extended to color images. They analyzed in more detail that the image stretched by the DCST features a higher local contrast image. From the saturation component, it's possible to estimate the transmission coefficient or the alpha map, and out of this, a haze-free image could be recovered that has the greater visibility than that of the captured hazy image. Fan, Xin et al. [18] presented a black channel prior-based transmission model that could explicitly formulates aerial perspective implying human perception on natural haze. They introduced 
maximum visibility as a parameter in to the transmission model, so they could naturally edit the quantity of haze in a picture by tuning this parameter with an actual interpretation. Additionally, they derived color correction and sky compensation from the transmission model, which improves the image quality for haze editing. Experimental results demonstrate the power of the proposed approach to generate images with various levels of haze in an all-natural and efficient manner. Comparisons with the standard algorithms on haze removal show the performance of the proposed algorithm when it comes to two objective metrics that evaluate the visibility and fidelity of the restored images. Ling, Zhigang et al. [19] proposed an efficient single dehazing algorithm via adaptive transmission compensation centered on human visual system (HVS). Finally, an adaptive transmission compensation strategy is presented to get rid of the haze and simultaneously suppress the halo artifacts or noise in the saturation areas. Experimental results indicate that proposed method can efficiently enhance the visibility of the foggy images in the challenging condition.

\section{PROPOSED METHODOLOGY}

Figure 1 shows the flowchart of the proposed methodology

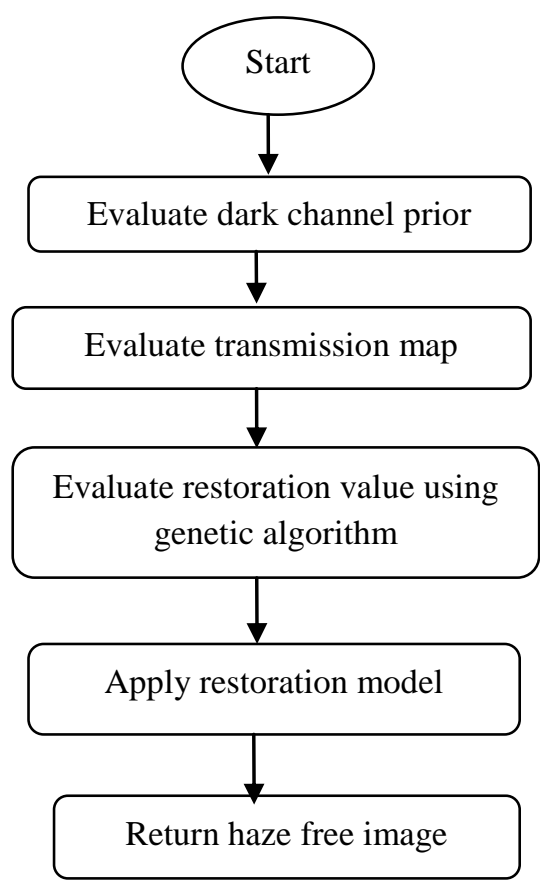

Fig 1: Flowchart of the proposed methodology

The steps for the proposed algorithm are :

Step 1: Input image

Step 2: Evaluate the dark channel prior of the input image.

Step 3: In this step, transmission map is evaluated.

Step 4: Here restoration value using genetic algorithm is evaluated.

Step 5: Then in this step, restoration model is applied.

Step 6: Here haze free image is returned.

\section{RESULTS AND DISCUSSIONS}

The proposed algorithm is tested on various images. The algorithm is applied using various performance indices accuracy, bit error rate, f-measure and specificity. In order to implement the proposed algorithm, design and implementation has been done in MATLAB using image processing toolbox. We are comparing proposed approach using some performance metrics

\section{EXPERIMENTAL SETUP}

Figure 2 represents the input image and the results of the existing technique and proposed image. From the figure it is clear that existing technique fails in case of images.
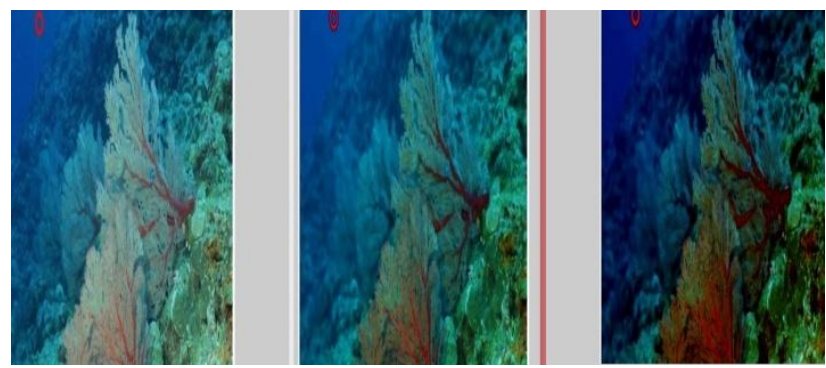

Fig 2 : Input Image and Results of Existing Technique and Proposed Technique

Figure 3 represents the input image and the results of the proposed technique on input image. From the figure it is clear that proposed technique gives efficient results in case of input image.

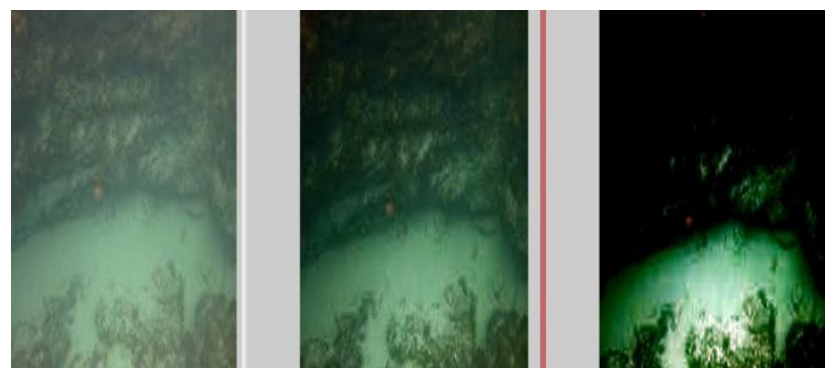

Fig 3: Input Image and Results of Existing Technique and Proposed Technique

\section{PERFORMANCE EVALUATION}

\subsection{MSE}

In image processing mean square error is the most general measure for performance measurement of the existing method and the coded images. It is computed by using equation

$$
\operatorname{MSE}=\frac{1}{M N} \sum_{i=1}^{M} \sum_{j=1}^{N}\left(f(i, j)-f^{\prime}(i, j)\right)^{2}
$$

Table 1 has shown the analysis of the mean square error. Mean Square Error needs to be less for the proposed algorithm for obtaining better results than existing technique. As shown in the table the results for proposed algorithm are less in every case. This shows the efficiency of proposed algorithm.

Table 1: MSE Evaluation

\begin{tabular}{|l|c|c|}
\hline Image & $\begin{array}{l}\text { Existing } \\
\text { Technique }\end{array}$ & $\begin{array}{l}\text { Proposed } \\
\text { Technique }\end{array}$ \\
\hline 1 & 154.9 & 108.1 \\
\hline 2 & 153.2 & 102.5 \\
\hline 3 & 154.1 & 123.2 \\
\hline 4 & 153.8 & 137.7 \\
\hline 5 & 153.9 & 124.06 \\
\hline
\end{tabular}




\begin{tabular}{|l|l|l|}
\hline 6 & 148.3 & 115.7 \\
\hline 7 & 154.4 & 109.8 \\
\hline 8 & 151.8 & 112.6 \\
\hline 9 & 154.7 & 128.6 \\
\hline 10 & 122.8 & 105.1 \\
\hline
\end{tabular}

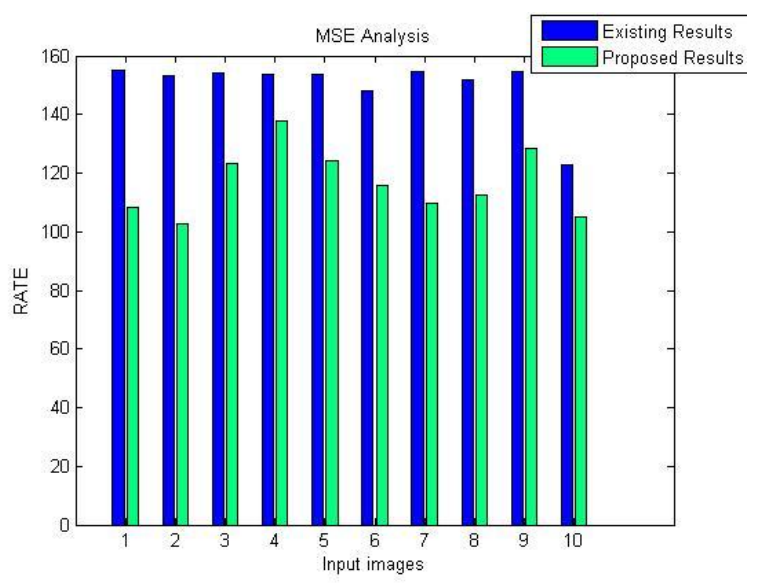

Fig 4: MSE Analysis

Figure 4 is showing the comparative analysis of the Mean Square Error. Mean Square Error needs to be less for the proposed algorithm for obtaining better results than existing technique. It can be seen clearly in the graph above, the results for proposed algorithm are less in every case as compared to existing technique. This shows the efficiency of proposed algorithm.

\subsection{PSNR}

The PSNR block computes the peak signal-to-noise ratio, between two images. This ratio is often used as a quality measurement between the original and a fused image. The higher the PSNR shows the better the quality of the fused or reconstructed image. PSNR value is computed by following equation:

$$
P S N R=10 \log _{10}\left(\frac{255^{2}}{M S E}\right)
$$

As PSNR need to be maximized; so the main goal is to increase the PSNR as much as possible.

Table 2 shows the comparable analysis of the Peak Signal to Noise Ratio (PSNR). PSNR needs to maximum for the proposed algorithm than existing techniques. As shown in the table the results for proposed algorithm are maximum in every case. Therefore proposed algorithm is providing better results than existing techniques.

Table 2 :PSNR Evaluation

\begin{tabular}{|l|l|l|}
\hline Image & $\begin{array}{l}\text { Existing } \\
\text { Technique }\end{array}$ & $\begin{array}{l}\text { Proposed } \\
\text { Technique }\end{array}$ \\
\hline 1 & 27.24 & 31.40 \\
\hline 2 & 18.19 & 23.93 \\
\hline 3 & 19.99 & 31.19 \\
\hline 4 & 23.49 & 35.21 \\
\hline 5 & 25.35 & 34.28 \\
\hline 6 & 42.48 & 45.66 \\
\hline
\end{tabular}

\begin{tabular}{|l|l|l|}
\hline 7 & 57.29 & 69.56 \\
\hline 8 & 18.26 & 23.23 \\
\hline 9 & 21.15 & 25.03 \\
\hline 10 & 36.23 & 23.40 \\
\hline
\end{tabular}

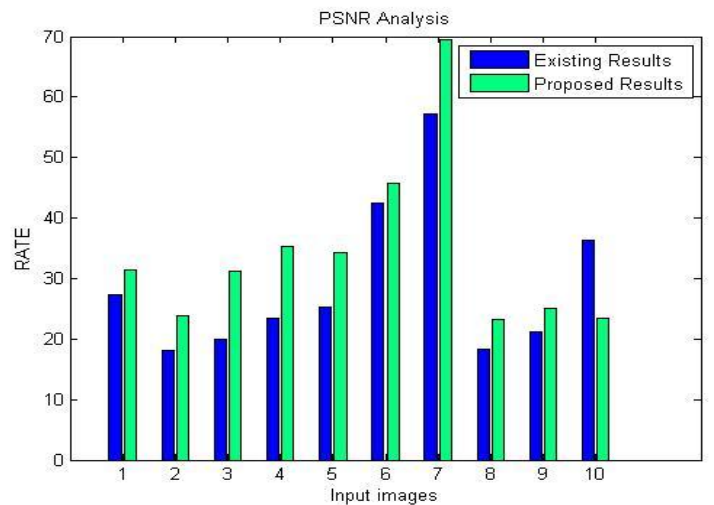

Fig 5: PSNR Analysis

Figure 5 is showing the comparative analysis of the Peak Signal to Noise ratio. Peak Signal to Noise ratio needs to be more for the proposed algorithm for obtaining better results than existing technique. It can be seen clearly in the graph above, the results for proposed algorithm are more in every case as compared to existing technique. This shows the efficiency of proposed algorithm.

ROOT MEAN SQUARE ERROR:- RMSE:RMSE is the measure of differences between values predicted by a model or an estimator and the values actually observed. Basically, the RMSE represents the sample standard deviation of the differences between input image and the final fog free image. Root mean square error is calculated by the given formulae

$$
\text { RMSE }=\frac{\Sigma\left(\mathrm{f}\left(\mathrm{x}_{\mathrm{i}}\right)-\mathrm{y}_{\mathrm{i}}\right)^{2}}{\mathrm{n}}
$$

Table 3 is showing the comparative analysis of the root mean square error. Table has clearly shown that is less in our case therefore the algorithm has shown significant results over the available algorithm.

Table 3: RMSE Evaluation

\begin{tabular}{|l|l|l|}
\hline Image & $\begin{array}{l}\text { Existing } \\
\text { Technique }\end{array}$ & $\begin{array}{l}\text { Proposed } \\
\text { Technique }\end{array}$ \\
\hline 1 & 0.2277 & 0.1515 \\
\hline 2 & 0.1724 & 0.1223 \\
\hline 3 & 0.1532 & 0.1462 \\
\hline 4 & 0.2146 & 0.1512 \\
\hline 5 & 0.1901 & 0.1503 \\
\hline 6 & 0.2742 & 0.1517 \\
\hline 7 & 0.2411 & 0.1713 \\
\hline 8 & 0.2444 & 0.1247 \\
\hline 9 & 0.1804 & 0.1363 \\
\hline 10 & 0.2337 & 0.1517 \\
\hline & & \\
\hline
\end{tabular}




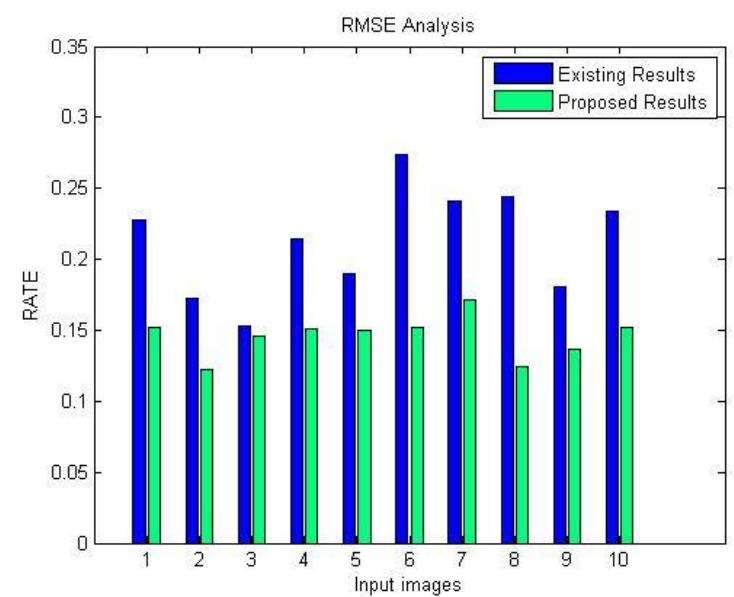

Figure 6: RMSE Evaluation

Figure 6 has shown the quantized analysis of the Root mean squared Error of different images. It is very clear from the plot that there is decrease in RMSE value of images with the use of proposed method over existing method. This decrease represents improvement in the objective quality of the image

Normalized Cross Correlation:- Table 4 has shown the comparison among proposed and the existing strategy based on Normalized Cross Correlation As the Normalized Cross Correlation is more in almost every taken road image; therefore the proposed strategy has shown significant results over the available technique.

Table 4: Normalized Cross Correlation Analysis

\begin{tabular}{|l|c|c|}
\hline IMAGES & $\begin{array}{c}\text { EXISTING } \\
\text { TECHNIQUE }\end{array}$ & $\begin{array}{l}\text { PROPOSED } \\
\text { TECHNIQUE }\end{array}$ \\
\hline 1 & 0.3934 & 0.511 \\
\hline 2 & 0.6399 & 0.7553 \\
\hline 3 & 0.8151 & 0.8188 \\
\hline 4 & 0.6962 & 0.7021 \\
\hline 5 & 0.5928 & 0.6152 \\
\hline 6 & 0.5851 & 0.5922 \\
\hline 7 & 0.7568 & 0.7771 \\
\hline 8 & 0.5653 & 0.6721 \\
\hline 9 & 0.3576 & 0.4169 \\
\hline 10 & 0.6124 & 0.6315 \\
\hline
\end{tabular}

Figure 7 has shown the comparison among proposed and the existing strategy based on Normalized Cross Correlation. As the Normalized Cross Correlation is more in almost every taken image; therefore the proposed strategy has shown significant results over the available technique.

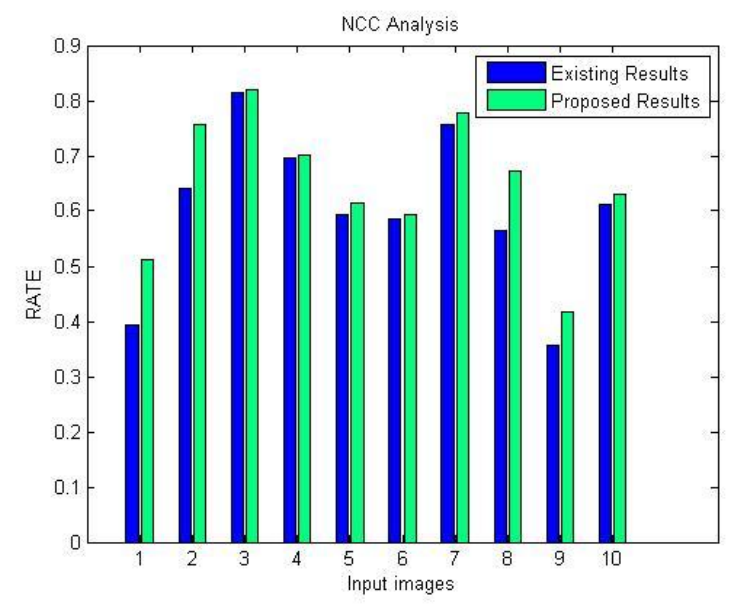

Fig. 7: Normalized Cross Correlation Analysis

\section{CONCLUSION AND FUTURE SCOPE}

In this paper, a new fog removal technique has been proposed which uses dark channel prior and genetic algorithm. The proposed algorithm is designed and implemented in MATLAB using image processing toolbox. The results produced by the existing dark channel prior method have less PSNR and NCC value and more RMSE and MSE. Therefore the overall objective is to improve the results by combining genetic algorithm with Dark channel prior method.

\section{ACKNOWLEDGMENTS}

I would like to thanks god, my family, my teachers, my friends to guide and support me to write this paper. They always help me when I need.

\section{REFERENCES}

[1] Podzimek, Josef. "Aerosol particle scavenging by fog and haze droplets."Studia Geophysica et Geodaetica 42, no. 4 (1998): 540-560.

[2] Chu, Chao-Tsung, and Ming-Sui Lee. "A contentadaptive method for single image dehazing." In Advances in Multimedia Information Processing-PCM 2010, pp. 350-361. Springer Berlin Heidelberg, 2010.

[3] Ancuti, Codruta O., Cosmin Ancuti, Chris Hermans, and Philippe Bekaert. "A fast semi-inverse approach to detect and remove the haze from a single image." In Computer Vision-ACCV 2010, pp. 501-514. Springer Berlin Heidelberg, 2011.

[4] Zhang, Yong-Qin, Yu Ding, Jin-Sheng Xiao, Jiaying Liu, and Zongming Guo. "Visibility enhancement using an image filtering approach." EURASIP Journal on Advances in Signal Processing 2012, no. 1 (2012): 1-6.

[5] Xiao, Chunxia, and Jiajia Gan. "Fast image dehazing using guided joint bilateral filter." The Visual Computer 28, no. 6-8 (2012): 713-721.

[6] Xie, Bin, Fan Guo, and Zixing Cai. "Fast Haze Removal Algorithm for Surveillance Video." In Measuring Technology and Mechatronics Automation in Electrical Engineering, pp. 235-241. Springer New York, 2012.

[7] Kim, Eun-Kyoung, Jae-Dong Lee, Byungin Moon, and Yong-Hwan Lee. "Hardware Architecture of Bilateral Filter to Remove Haze." In Communication and Networking, pp. 129-135. Springer Berlin Heidelberg, 2012. 
[8] Ding, Meng, and RuoFeng Tong. "Efficient dark channel based image dehazing using quadtrees." Science China Information Sciences 56, no. 9 (2013): 1-9.

[9] Xue, Yungang, Ju Ren, Huayou Su, Mei Wen, and Chunyuan Zhang. "Parallel Implementation and Optimization of Haze Removal Using Dark Channel Prior Based on CUDA." In High Performance Computing, pp. 99-109. Springer Berlin Heidelberg, 2013.

[10] Lan, Xia, Liangpei Zhang, Huanfeng Shen, Qiangqiang Yuan, and Huifang Li. "Single image haze removal considering sensor blur and noise." EURASIP Journal on Advances in Signal Processing 2013, no. 1 (2013): 1-13.

[11] Ogorodnikov, B. I., and V. E. Khan. "Impact of haze and fog on filter characteristics in the process of monitoring radioactive aerosol." Russian Meteorology and Hydrology 38, no. 11 (2013): 787-791.

[12] Guo, Fan, Jin Tang, and Zi-Xing Cai. "Image dehazing based on haziness analysis." International Journal of Automation and Computing 11, no. 1 (2014): 78-86.

[13] Galdran, Adrian, Javier Vazquez-Corral, David Pardo, and Marcelo Bertalmío. "A Variational Framework for Single Image Dehazing." In Computer Vision-ECCV
2014 Workshops, pp. 259-270. Springer International Publishing, 2014.

[14] Zhang, Jun, and Shiqiang Hu. "A GPU-accelerated realtime single image de-hazing method using pixel-level optimal de-hazing criterion." Journal of Real-Time Image Processing 9, no. 4 (2014): 661-672.

[15] Liu, Qian, MaoYin Chen, and DongHua Zhou. "Single image haze removal via depth-based contrast stretching transform." Science China Information Sciences: 1-17. Volume 58, Issue 1, pp 1-17, 2014

[16] Gadnayak, Khitish Kumar, Pankajini Panda, and Niranjan Panda. "Haze Removal: An Approach Based on Saturation Component." In Intelligent Computing, Communication and Devices, pp. 281-287. Springer India, 2015.

[17] Fan, Xin, Yi Wang, Renjie Gao, and Zhongxuan Luo. "Haze editing with natural transmission." The Visual Computer (2015): 1-11.

[18] Ling, Zhigang, Shutao Li, Yaonan Wang, He Shen, and Xiao Lu. "Adaptive transmission compensation via human visual system for efficient single image dehazing." The Visual Computer (2015): 1-10. 